\title{
Preferential hydration of lysozyme in water/glycerol mixtures: A small-angle neutron scattering study
}

Raffaele Sinibaldi, Maria Grazia Ortore, Francesco Spinozzi, Flavio Carsughi, Henrich Frielinghaus, Stefania Cinelli, Giuseppe Onori, and Paolo Mariani

Citation: The Journal of Chemical Physics 126, 235101 (2007); doi: 10.1063/1.2735620

View online: https://doi.org/10.1063/1.2735620

View Table of Contents: http://aip.scitation.org/toc/jcp/126/23

Published by the American Institute of Physics

\section{Articles you may be interested in}

Microcalorimetric study of thermal unfolding of lysozyme in water/glycerol mixtures: An analysis by solvent exchange model

The Journal of Chemical Physics 129, 035101 (2008); 10.1063/1.2945303

Comparison of simple potential functions for simulating liquid water

The Journal of Chemical Physics 79, 926 (1983); 10.1063/1.445869

Communication: Contrasting effects of glycerol and DMSO on lipid membrane surface hydration dynamics and forces

The Journal of Chemical Physics 145, 041101 (2016); 10.1063/1.4959904

The Kirkwood-Buff theory and the effect of cosolvents on biochemical reactions

The Journal of Chemical Physics 121, 9147 (2004); 10.1063/1.1806402

The Statistical Mechanical Theory of Solutions. I

The Journal of Chemical Physics 19, 774 (1951); 10.1063/1.1748352

Preferential solvation of lysozyme in water/ethanol mixtures

The Journal of Chemical Physics 135, 245103 (2011); 10.1063/1.3670419

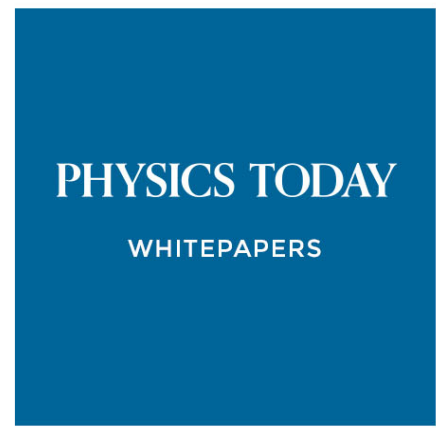

ADVANCED LIGHT CURE ADHESIVES

Take a closer look at what these environmentally friendly adhesive systems can do

\section{READ NOW}

PRESENTED BY

Q MASTERBOND' 


\title{
Preferential hydration of lysozyme in water/glycerol mixtures: A small-angle neutron scattering study
}

\author{
Raffaele Sinibaldi, ${ }^{\text {a) }}$ Maria Grazia Ortore, and Francesco Spinozzi \\ Dipartimento di Scienze Applicate ai Sistemi Complessi, Università Politecnica delle Marche, \\ Ancona 60131, Italy \\ Flavio Carsughi \\ Dipartimento di Scienze Applicate ai Sistemi Complessi, Università Politecnica delle Marche, \\ Ancona 60131, Italy and Institut für Festkörperforschung, Forschungszentrum, Julich 52425, Germany \\ Henrich Frielinghaus \\ Institut für Festkörperforschung, Forschungszentrum, Julich 52425, Germany \\ Stefania Cinelli and Giuseppe Onori \\ Dipartimento di Fisica, Università di Perugia, Centro di Eccelenza Materiali Innovativi Nanostrutturati, \\ INFM-CRS SOFT, Perugia I-06123, Italy \\ Paolo Mariani \\ Dipartimento di Scienze Applicate ai Sistemi Complessi, Università Politecnica delle Marche, \\ Ancona 60131, Italy
}

(Received 12 January 2007; accepted 5 April 2007; published online 15 June 2007)

\begin{abstract}
In solution small-angle neutron scattering has been used to study the solvation properties of lysozyme dissolved in water/glycerol mixtures. To detect the characteristics of the protein-solvent interface, 35 different experimental conditions (i.e., protein concentration, water/glycerol fraction in the solvent, content of deuterated compounds) have been considered and a suitable software has been developed to fit simultaneously the whole set of scattering data. The average composition of the solvent in the close vicinity of the protein surface at each experimental condition has been derived. In all the investigated conditions, glycerol resulted especially excluded from the protein surface, confirming that lysozyme is preferentially hydrated. By considering a thermodynamic hydration model based on an equilibrium exchange between water and glycerol from the solvation layer to the bulk, the preferential binding coefficient and the excess solvation number have been estimated. Results were compared with data previously derived for ribonuclease $A$ in the same mixed solvent: even if the investigated solvent compositions were very different, the agreement between data is noticeable, suggesting that a unique mechanism presides over the preferential hydration process. Moreover, the curve describing the excess solvation number as a function of the solvent composition shows the occurrence of a region of maximal hydration, which probably accounts for the changes in protein stability detected in the presence of cosolvents. (C) 2007 American Institute of Physics. [DOI: 10.1063/1.2735620]
\end{abstract}

\section{INTRODUCTION}

The physicochemical properties of proteins in solution markedly depend on the role of water in various noncovalent interactions, including solvation of ionic groups and dipoles, hydrogen bonding, and hydrophobic interactions. ${ }^{1,2}$ As a consequence, denaturating or stabilizing effects have been observed in the presence of cosolvents. In particular, sugars and polyols are well known to stabilize proteins against chemical or physical denaturation, even if the molecular mechanism regulating such a process is still an argument of scientific investigation. ${ }^{3-5}$

Indeed, glycerol/water mixtures have been extensively studied. Early studies suggested that, in the presence of glyc-

\footnotetext{
a) Author to whom correspondence should be addressed. Present address: Dipartimento di Scienze Applicate ai Sistemi Complessi, Università Politecnica delle Marche, Via Brecce Bianche, Ancona I-60131, Italy. Tel.: (+39)071-2204607; Fax: (+39)071-2204605.
}

erol, water molecules form coating shells around proteins ${ }^{6,7}$ and that polyols and sugars are preferentially excluded from the protein surface. ${ }^{8}$ Moreover, small amounts of water added to a protein solution of pure glycerol appeared preferentially driven to the protein surface. ${ }^{9}$

From a biological point of view, the case of lysozyme in glycerol is particularly relevant: two-dimensional ${ }^{1} \mathrm{H}-\mathrm{NMR}$ and circular dichroism spectroscopy studies revealed that secondary and tertiary structures of lysozyme in glycerol are similar to those observed in water, ${ }^{10}$ while protein powders may exhibit reversible changes in their secondary structure. ${ }^{11}$ In addition, the unfolded enzyme was found to spontaneously regain substantial catalytic activity even in the nearly anhydrous glycerol, with an efficiency comparable with that in aqueous solutions. ${ }^{12}$

Some of us have recently studied the influence of hydration on the internal dynamics of lysozyme embedded in glycerol matrix by neutron scattering. ${ }^{13,14}$ Results provide a co- 
herent description of a hydration-dependent dynamics of the protein, which is activated above a threshold water concentration in the solvent, while it shows a saturation effect at high water content. It was suggested that water molecules activate the whole lysozyme dynamics by progressively hydrating the protein surface groups, playing the role of plasticizer through a preferential hydration effect. More recently, a calorimetric study has shown that the addition of small amounts of water in a lysozyme-glycerol matrix modifies the melting behavior of the protein: on increasing the quantity of water, the melting temperature of lysozyme decreases, suggesting an inverse correlation between protein stability and structure flexibility. ${ }^{15}$

Understanding the role played by hydration in activating protein intrinsic dynamics, as well as the link between preferential hydration, structural rearranging, and biological functionality, requires a direct characterization of the proteinsolvent interface. However, due to local modifications, complex heterogeneity, sensitivity requirement, and composition modification in mixed solvent, the structural analysis of the solvation shell appears experimentally very problematic. Until a few years ago, even molecular dynamic simulations were sparingly applied in this field. ${ }^{5,16,17}$

Small-angle x-ray and neutron scattering (SAXS and SANS) methods are very suitable techniques to derive information on the structural properties of proteins in solution. Indeed, by SANS contrast variation, or combining SAXS and SANS, heterogeneous macromolecular systems, such as protein-nucleic acid complexes and protein-lipid systems, have been fully characterized. ${ }^{18-21}$ Particularly relevant for the present work are results obtained comparing SAXS and SANS data from proteins dissolved in mixtures of light and heavy waters, which have proven that water density at the protein surface is significantly higher than in the bulk. ${ }^{7,22}$ The different scattering contrasts expected between protein, solution, and solvation shells should then make the quantitative analysis of the protein hydration shell possible by SANS. In this work, we have applied SANS contrast variation technique on lysozyme dissolved in water/glycerol mixtures. As differences in composition between the solvation shell and solution were expected to be quite small, the investigated experimental conditions were selected on the basis of extended numerical simulations, while the whole experimental data were analyzed using a global fit, an approach that we demonstrated to be very efficient for extracting tiny structural details from a wide experimental context. ${ }^{23,24}$ Accordingly, the solvent composition of the local domain was derived and, considering a thermodynamic hydration model based on an equilibrium exchange between water and glycerol from the solvation layer to the bulk, the preferential binding coefficient and the excess solvation number were estimated.

\section{MATERIALS AND METHODS}

\section{A. Samples preparation}

Protein solutions at weight concentrations $c$ of 30,50 , $90,110,130$, and $150 \mathrm{~g} \mathrm{l}^{-1}$ were prepared by dissolving the requested amount of lysozyme powder (hen egg white lysozyme, 99\% purity, Sigma Aldrich) in water/glycerol mixtures of different compositions and of different deuteration grades. Mixed solutions were prepared using pure light and heavy waters and hydrogenated and deuterated glycerol (all from Sigma Aldrich). To obtain similar $p \mathrm{H}$ conditions, $\mathrm{HCl}$ $1 M$ was added to the mixed solutions: the amount of added $\mathrm{HCl}$ was previously determined by adjusting water solutions of lysozyme at all investigated concentrations $c$ to $p \mathrm{H}$ $=4.5 \pm 0.1$. To avoid uncertainties due to hydrogen-deuterium exchange, the same amount of deuterated water and glycerol was used to prepare the solution at the requested deuteration grade, i.e., $x_{\mathrm{D}}=n_{w, \mathrm{D}} /\left(n_{w, \mathrm{H}}+n_{w, \mathrm{D}}\right) \equiv n_{g, \mathrm{D}} /\left(n_{g, \mathrm{H}}+n_{g, \mathrm{D}}\right)$, where $n_{w, \mathrm{D}}, n_{w, \mathrm{H}}$, and $n_{g, \mathrm{D}}, n_{g, \mathrm{H}}$ are the number densities (number of molecules per total volume) of heavy and light waters and deuterated and hydrogenated glycerols, respectively. Three $x_{\mathrm{D}}$ conditions were analyzed, namely, $0,0.05$, and 0.35 . The investigated water molar fraction in the solvent, $x_{w}=n_{w} /\left(n_{w}\right.$ $+n_{g}$ ) (with $n_{w}=n_{w, \mathrm{H}}+n_{w, \mathrm{D}}$ and $n_{g}=n_{g, \mathrm{H}}+n_{g, \mathrm{D}}$ ), ranges from 0.4 to 0.6 , which, for a protein concentration $c=90 \mathrm{~g} \mathrm{l}^{-1}$, corresponds to a water-to-protein weight ratio ranging from 1 to 3 . According to the considered $c, x_{w}$, and $x_{\mathrm{D}}$, the 35 different experimental conditions listed in Table I were then investigated.

\section{B. SANS experiments}

Measurements were performed at Forschungerszentrum (FZJ) in Jülich (Germany) using the KWS1 diffractometer. Samples were measured at room temperature in $1 \mathrm{~mm}$ thick quartz cells using a neutron wavelength $\lambda=6 \AA$ and a sample detector distance of $1.25 \mathrm{~m}$. The investigated scattering vector $Q$ ranges between 0.05 and $0.28 \AA^{-1}(Q=4 \pi \sin \theta / \lambda$ being $2 \theta$ the scattering angle). Radially averaged scattered intensities were corrected for background, buffer contribution, detector inhomogeneities, and sample transmission, and were converted in absolute units $\left(\mathrm{cm}^{-1}\right)$ by calibration with $1 \mathrm{~mm}$ of light water.

\section{SANS THEORY FOR PROTEINS IN A BINARY SOLVENT}

The macroscopical differential neutron scattering cross section for monodisperse and randomly oriented protein particles dissolved in a solvent is ${ }^{18}$

$$
\frac{d \Sigma}{d \Omega}(Q)=n_{p} S(Q) P(Q)+B,
$$

where $n_{p} \equiv c N_{A} / M_{p}$ is the protein number density $\left(N_{A}\right.$ is Avogadro's number and $M_{p}$ the protein molecular weight), $S(Q)$ is the effective structure factor, $P(Q)$ is the protein averaged squared form factor, and $B$ is a flat background.

$S(Q)$ has been modeled under the random phase approximation, as described in Ref. 25. Here, we point out that the 
TABLE I. Experimental compositions (first column) and optimized parameters (model 1 and model 2) for all investigated samples by global fitting procedure. The symbols and abbreviations as in the text. For both models, the error on $x_{w, l}$ is found to be 0.02 . Errors on sample composition are less than $0.5 \%$.

\begin{tabular}{|c|c|c|c|c|c|c|c|c|c|}
\hline \multirow[b]{2}{*}{$\begin{array}{c}c \\
\left(\mathrm{~g} \mathrm{l^{-1 }}\right)\end{array}$} & \multirow[b]{2}{*}{$x_{D}$} & \multirow[b]{2}{*}{$x_{w}$} & \multicolumn{4}{|c|}{ Model 1} & \multicolumn{3}{|c|}{ Model 2} \\
\hline & & & $\begin{array}{c}B \\
\left(10^{-2} \mathrm{~cm}^{-1}\right)\end{array}$ & $\begin{array}{c}J \\
\left(k_{B} T\right)\end{array}$ & $\begin{array}{c}d \\
(\AA)\end{array}$ & $x_{w, l}$ & $\begin{array}{c}B \\
\left(10^{-2} \mathrm{~cm}^{-1}\right)\end{array}$ & $\begin{array}{c}J \\
\left(k_{B} T\right)\end{array}$ & $\begin{array}{c}d \\
(\AA)\end{array}$ \\
\hline \multirow[t]{3}{*}{30} & & & \multicolumn{4}{|c|}{$I_{S}=21 \pm 3 \mathrm{mM}$} & \multicolumn{3}{|c|}{$I_{S}=32 \pm 4 \mathrm{mM}$} \\
\hline & 0 & 0.46 & $2.38 \pm 0.03$ & $2.3 \pm 0.1$ & $5.7 \pm 0.3$ & 0.64 & $2.39 \pm 0.02$ & $2.36 \pm 0.09$ & $5.2 \pm 0.2$ \\
\hline & 0 & 0.56 & $2.36 \pm 0.03$ & $3.2 \pm 0.1$ & $3.3 \pm 0.3$ & 0.51 & $2.35 \pm 0.02$ & $3.74 \pm 0.09$ & $2.7 \pm 0.2$ \\
\hline \multirow[t]{3}{*}{50} & & & \multicolumn{4}{|c|}{$I_{S}=38 \pm 8 \mathrm{mM}$} & \multicolumn{3}{|c|}{$I_{S}=53 \pm 2 \mathrm{mM}$} \\
\hline & 0 & 0.46 & $4.05 \pm 0.02$ & $2.3 \pm 0.1$ & $5.8 \pm 0.3$ & 0.69 & $3.95 \pm 0.01$ & $2.34 \pm 0.09$ & $5.2 \pm 0.2$ \\
\hline & 0 & 0.56 & $3.80 \pm 0.02$ & $3.2 \pm 0.1$ & $3.3 \pm 0.3$ & 0.62 & $3.78 \pm 0.02$ & $3.74 \pm 0.09$ & $2.7 \pm 0.2$ \\
\hline \multirow[t]{6}{*}{90} & & & \multicolumn{4}{|c|}{$I_{S}=60 \pm 2 \mathrm{mM}$} & \multicolumn{3}{|c|}{$I_{S}=62 \pm 3 \mathrm{mM}$} \\
\hline & 0 & 0.46 & $7.50 \pm 0.02$ & $2.3 \pm 0.1$ & $5.8 \pm 0.3$ & 0.51 & $7.42 \pm 0.02$ & $2.32 \pm 0.09$ & $5.2 \pm 0.2$ \\
\hline & 0 & 0.49 & $7.30 \pm 0.03$ & $2.62 \pm 0.09$ & $5.0 \pm 0.3$ & 0.70 & $7.35 \pm 0.02$ & $2.80 \pm 0.09$ & $4.4 \pm 0.2$ \\
\hline & 0 & 0.51 & $7.15 \pm 0.02$ & $2.74 \pm 0.09$ & $4.6 \pm 0.3$ & 0.62 & $7.20 \pm 0.03$ & $3.00 \pm 0.09$ & $4.0 \pm 0.2$ \\
\hline & 0 & 0.56 & $7.00 \pm 0.02$ & $3.2 \pm 0.1$ & $3.3 \pm 0.3$ & 0.56 & $6.92 \pm 0.02$ & $3.72 \pm 0.09$ & $2.7 \pm 0.2$ \\
\hline & 0 & 0.57 & $7.00 \pm 0.03$ & $3.2 \pm 0.1$ & $3.2 \pm 0.3$ & 0.65 & $7.05 \pm 0.02$ & $3.79 \pm 0.09$ & $2.6 \pm 0.2$ \\
\hline \multirow[t]{10}{*}{110} & & & \multicolumn{4}{|c|}{$I_{S}=73 \pm 2 \mathrm{mM}$} & \multicolumn{3}{|c|}{$I_{S}=72 \pm 2 \mathrm{mM}$} \\
\hline & 0 & 0.41 & $9.00 \pm 0.03$ & $1.8 \pm 0.1$ & $7.2 \pm 0.3$ & 0.69 & $9.05 \pm 0.03$ & $1.5 \pm 0.1$ & $6.6 \pm 0.2$ \\
\hline & 0 & 0.46 & $8.78 \pm 0.02$ & $2.3 \pm 0.1$ & $5.8 \pm 0.3$ & 0.73 & $8.75 \pm 0.02$ & $2.35 \pm 0.09$ & $5.2 \pm 0.2$ \\
\hline & 0 & 0.47 & $8.72 \pm 0.03$ & $2.4 \pm 0.1$ & $5.6 \pm 0.3$ & 0.61 & $8.75 \pm 0.02$ & $2.43 \pm 0.09$ & $5.0 \pm 0.2$ \\
\hline & 0 & 0.52 & $8.60 \pm 0.04$ & $2.9 \pm 0.1$ & $4.2 \pm 0.3$ & 0.70 & $8.62 \pm 0.02$ & $3.24 \pm 0.09$ & $3.6 \pm 0.2$ \\
\hline & 0 & 0.56 & $8.42 \pm 0.05$ & $3.2 \pm 0.1$ & $3.2 \pm 0.3$ & 0.67 & $8.40 \pm 0.02$ & $3.79 \pm 0.09$ & $2.6 \pm 0.2$ \\
\hline & 0 & 0.57 & $8.40 \pm 0.03$ & $3.3 \pm 0.1$ & $3.1 \pm 0.3$ & 0.73 & $8.32 \pm 0.02$ & $3.84 \pm 0.09$ & $2.5 \pm 0.2$ \\
\hline & 0 & 0.58 & $8.40 \pm 0.03$ & $3.4 \pm 0.1$ & $2.8 \pm 0.3$ & 0.62 & $8.42 \pm 0.03$ & $3.98 \pm 0.09$ & $2.3 \pm 0.2$ \\
\hline & 0.05 & 0.56 & $8.20 \pm 0.01$ & $1.8 \pm 0.1$ & $9.9 \pm 0.8$ & 0.66 & $8.15 \pm 0.02$ & $1.9 \pm 0.2$ & $8.9 \pm 0.3$ \\
\hline & 0.35 & 0.56 & $5.24 \pm 0.02$ & $2.3 \pm 0.5$ & $0.71 \pm 0.07$ & 0.68 & $5.10 \pm 0.11$ & $1.6 \pm 0.5$ & $0.6 \pm 0.1$ \\
\hline \multirow[t]{11}{*}{130} & & & \multicolumn{4}{|c|}{$I_{S}=81.9 \pm 0.5 \mathrm{mM}$} & \multicolumn{3}{|c|}{$I_{S}=78 \pm 3 \mathrm{mM}$} \\
\hline & 0 & 0.41 & $10.50 \pm 0.04$ & $1.8 \pm 0.1$ & $7.2 \pm 0.3$ & 0.67 & $10.40 \pm 0.10$ & $1.6 \pm 0.1$ & $6.6 \pm 0.2$ \\
\hline & 0 & 0.46 & $10.20 \pm 0.05$ & $2.3 \pm 0.1$ & $5.8 \pm 0.3$ & 0.66 & $10.25 \pm 0.05$ & $2.32 \pm 0.09$ & $5.2 \pm 0.2$ \\
\hline & 0 & 0.47 & $10.15 \pm 0.05$ & $2.3 \pm 0.1$ & $5.8 \pm 0.3$ & 0.69 & $10.10 \pm 0.05$ & $2.33 \pm 0.09$ & $5.2 \pm 0.2$ \\
\hline & 0 & 0.53 & $10.00 \pm 0.05$ & $2.9 \pm 0.1$ & $4.1 \pm 0.3$ & 0.61 & $10.05 \pm 0.05$ & $3.28 \pm 0.09$ & $3.5 \pm 0.2$ \\
\hline & 0 & 0.56 & $9.92 \pm 0.05$ & $3.2 \pm 0.1$ & $3.2 \pm 0.3$ & 0.62 & $9.87 \pm 0.05$ & $3.80 \pm 0.09$ & $2.6 \pm 0.2$ \\
\hline & 0 & 0.59 & $9.82 \pm 0.05$ & $3.4 \pm 0.1$ & $2.6 \pm 0.3$ & 0.68 & $9.85 \pm 0.05$ & $4.11 \pm 0.09$ & $2.0 \pm 0.2$ \\
\hline & 0.05 & 0.53 & $9.48 \pm 0.05$ & $1.7 \pm 0.1$ & $9.9 \pm 0.8$ & 0.62 & $9.40 \pm 0.05$ & $1.9 \pm 0.2$ & $8.9 \pm 0.3$ \\
\hline & 0.05 & 0.59 & $9.32 \pm 0.03$ & $1.8 \pm 0.1$ & $9.9 \pm 0.8$ & 0.56 & $9.36 \pm 0.03$ & $1.9 \pm 0.2$ & $8.9 \pm 0.3$ \\
\hline & 0.35 & 0.53 & $6.20 \pm 0.03$ & $2.2 \pm 0.4$ & $0.71 \pm 0.07$ & 0.62 & $6.25 \pm 0.05$ & $1.5 \pm 0.5$ & $0.6 \pm 0.1$ \\
\hline & 0.35 & 0.58 & $6.10 \pm 0.02$ & $2.3 \pm 0.5$ & $0.71 \pm 0.07$ & 0.67 & $6.05 \pm 0.05$ & $1.6 \pm 0.5$ & $0.6 \pm 0.1$ \\
\hline \multirow[t]{8}{*}{150} & & & \multicolumn{4}{|c|}{$I_{S}=98 \pm 2 \mathrm{mM}$} & \multicolumn{3}{|c|}{$I_{S}=107 \pm 3 \mathrm{mM}$} \\
\hline & 0 & 0.45 & $11.92 \pm 0.06$ & $2.2 \pm 0.1$ & $6.0 \pm 0.3$ & 0.65 & $11.86 \pm 0.05$ & $2.19 \pm 0.09$ & $5.5 \pm 0.2$ \\
\hline & 0 & 0.51 & $11.72 \pm 0.05$ & $2.75 \pm 0.09$ & $4.6 \pm 0.3$ & 0.67 & $11.75 \pm 0.03$ & $3.01 \pm 0.09$ & $4.0 \pm 0.2$ \\
\hline & 0 & 0.56 & $11.55 \pm 0.05$ & $3.2 \pm 0.1$ & $3.2 \pm 0.3$ & 0.68 & $11.49 \pm 0.03$ & $3.80 \pm 0.09$ & $2.6 \pm 0.2$ \\
\hline & 0 & 0.58 & $11.50 \pm 0.05$ & $3.4 \pm 0.1$ & $2.8 \pm 0.3$ & 0.58 & $11.53 \pm 0.03$ & $4.00 \pm 0.09$ & $2.2 \pm 0.2$ \\
\hline & 0.05 & 0.58 & $11.10 \pm 0.02$ & $1.8 \pm 0.1$ & $9.9 \pm 0.8$ & 0.60 & $11.07 \pm 0.03$ & $1.9 \pm 0.2$ & $8.9 \pm 0.3$ \\
\hline & 0.35 & 0.51 & $7.49 \pm 0.03$ & $2.2 \pm 0.4$ & $0.71 \pm 0.07$ & 0.65 & $7.45 \pm 0.03$ & $1.5 \pm 0.5$ & $0.6 \pm 0.1$ \\
\hline & 0.35 & 0.58 & $7.20 \pm 0.03$ & $2.3 \pm 0.5$ & $0.71 \pm 0.07$ & 0.58 & $7.24 \pm 0.03$ & $1.6 \pm 0.5$ & $0.6 \pm 0.1$ \\
\hline
\end{tabular}

two body interaction energy $u(r)$ has been written as

$$
u(r)=u_{\mathrm{HS}}(r)+u_{C}(r)+u_{A}(r),
$$

where $u_{\mathrm{HS}}(r)$ is the hard sphere potential (which depends on the protein diameter $\sigma), u_{C}(r)$ the Coulombic screened potential (which depends on the number of charge per protein $Z$, on the dielectric constant of the medium $\varepsilon$, and on the ionic strength of the solvent $\left.I_{S}\right)$, and $u_{A}(r)$ an attractive potential written as

$$
u_{A}(r)=-J \sigma \frac{\exp [-(r-\sigma) / d]}{r},
$$

where $J$ is the depth of the potential at contact $(r=\sigma)$ and $d$ its range.

The protein form factor in Eq. (1) should explicitly refer to solvated particles. Therefore, lysozyme in solution has been described as a particle of homogeneous scattering density (corresponding to the core protein domain $p$ ), covered by a shell of different scattering density (corresponding to the 
solvation layer, called local domain $l$ ), which can include more than one hydration layer. Indicating with $b$ the bulk solvent domain, the $P(Q)$ has been written as ${ }^{18-20}$

$$
\begin{aligned}
P(Q)= & \left(\rho_{p}-\rho_{b}\right)^{2} V_{p}^{2} P_{p p}(Q)+\left(\rho_{l}-\rho_{b}\right)^{2} V_{l}^{2} P_{l l}(Q)+2\left(\rho_{p}\right. \\
& \left.-\rho_{b}\right)\left(\rho_{l}-\rho_{b}\right) V_{p} V_{l} P_{p l}(Q)
\end{aligned}
$$

where $\rho_{p}, \rho_{i}$, and $\rho_{b}$ are the scattering length densities of the different domains, $V_{p}$ and $V_{l}$ are the scattering volumes of the protein molecule and of the local domain, and $P_{i j}(Q)$ is the partial form factor. ${ }^{19,20}$

As the lysozyme solutions have been prepared at different deuteration grades and at different water compositions, scattering length densities are different at each experimental condition. The scattering length density of lysozyme, $\rho_{p}$, has been determined from the amino acid composition, considering the exchangeable hydrogen atoms present in the molecule (see Ref. 21). The scattering length densities of the bulk and of the local domain have been written as

$$
\rho_{i}=\frac{x_{w, i}\left(a_{w}-a_{g}\right)+a_{g}}{x_{w, i}\left(\nu_{w, i}-\nu_{g, i}\right)+\nu_{g, i}} \quad \text { with } i=b, l,
$$

where $x_{w, i}$ is the water molar fraction in the bulk phase ( $i$ $=b)$ and in the local domain $(i=l), \nu_{w, i}$ and $\nu_{g, i}$ are partial molecular volumes of water and glycerol in the two domains, and $a_{w}$ and $a_{g}$ are the scattering lengths of water and glycerol at the considered $x_{\mathrm{D}}$. Parameters in Eq. (5) need to be further detailed: $x_{w, i}$ is equal to $n_{w, i} /\left(n_{w, i}+n_{g, i}\right)$, being $n_{w, i}$ and $n_{g, i}$ the number densities of water and of glycerol in the $i$ th domain (note that the mass balance condition imposes $n_{w}$ $=n_{w, b}+n_{w, l}$ and $\left.n_{g}=n_{g, b}+n_{g, l}\right) ; a_{w}$ and $a_{g}$ have been derived from atomic coherent scattering length data; ${ }^{21,26}$ values of $\nu_{g, b}$ and $\nu_{w, b}$ have been calculated as a function of $x_{w, b}$ from density data of water/glycerol mixtures reported in Ref. 27. Note that, at each solvent composition, $\nu_{g, l}$ has been considered equal to $\nu_{g, b}$, as no large variations of the steric hindrance of glycerol after the protein binding are expected. On the contrary, the molecular volume of water in the local domain has been allowed to be different from values calculated in the bulk because of electrostriction effects at the protein surface. $^{22}$ The protein and local domain volumes $\left(V_{p}\right.$ and $\left.V_{l}\right)$, as well as the partial form factors $P_{i j}(Q)$, have been calculated by the Monte Carlo method previously described ${ }^{19,20}$ on the basis of the lysozyme protein data bank (PDB) structure (entry 6LYZ) (Ref. 28) and considering a variable thickness of the local domain ( $\delta_{l}$, see below). Eventual variations in protein volume have been assumed to correspond to isotropic changes of the protein shape: if $V_{p}$ changes of a factor $\gamma$, the corresponding modifications of $P_{i j}(Q)$ result in a simple scaling on the $Q$ axis of a factor $Q \gamma^{1 / 3} \cdot{ }^{29}$ As the composition of the local domain is expected to be different from the composition of the bulk solvent, a last equation describing the dependence of $x_{w, b}$ on $x_{w}$ and on $x_{w, l}$ should be reported,

$$
x_{w, b}=\frac{\left[1-n_{p}\left(V_{p}+V_{l}\right)\right]\left[x_{w, l}\left(\nu_{g}-\nu_{w, l}\right)-\nu_{g}\right] x_{w}-n_{p} V_{l} \nu_{g}\left(x_{w}-x_{w, l}\right)}{\left[1-n_{p}\left(V_{p}+V_{l}\right)\right]\left[x_{w, l}\left(\nu_{g}-\nu_{w, l}\right)-\nu_{g}\right]+n_{p} V_{l}\left(\nu_{w, b}-\nu_{g}\right)\left(x_{w}-x_{w, l}\right)} .
$$

\section{A. Simulated experiments}

The experimental conditions described before (see Table I) were selected after numerical simulations of the form factor of lysozyme in different solvation states (e.g., $x_{w, l} / x_{w}$ from 0.8 to 1.4). Calculations were performed assuming a thickness for the solvation layer of $4.5 \AA$, i.e., larger than a single water layer, as recently demonstrated in water/glycerol mixtures. ${ }^{30,31}$

Some significative $n_{p} P(Q)$ profiles are shown in Fig. 1 . Results clearly indicate that a contrast variation series $(0.0$ $\left.\leqslant x_{\mathrm{D}} \leqslant 0.4\right)$ on samples at protein concentration around $100 \mathrm{~g}^{-1}$ should make the characterization of the solvation layer at different solvent compositions possible. It is important to point out that in all cases, the sensitivity of SANS to the difference in the scattering length density of the local domain compared to the bulk phase is quite small, as evidenced in Fig. 1 by solid, dotted, and dashed lines referring to different solvation states; however, these small contributions analyzed on a large number of samples would make this effect measurable.

\section{RESULTS AND DISCUSSION}

\section{A. Experimental Results}

SANS experimental curves are shown in Fig. 2, ordered according to the protein concentration $(c)$, to the water molar


FIG. 1. Simulated form factors for lysozyme in water/glycerol mixtures at different protein concentrations $\left(c\right.$, in $\mathrm{g}^{-1}$ ), solvent and local domain compositions ( $x_{w}$ and $x_{w, l}$, respectively), and deuteration grade of the solvent $\left(x_{\mathrm{D}}\right)$, as indicated. Left frame: $x_{\mathrm{D}}=0$, water/protein weight ratios equal to 1 . Right frame: $c=80 \mathrm{~g}^{-1}$ and $x_{w}=0.37$. The solid, dotted, and dashed lines refer to $x_{w, l} / x_{w}$ ratios of $1,1.2$, and 1.4 , respectively. 


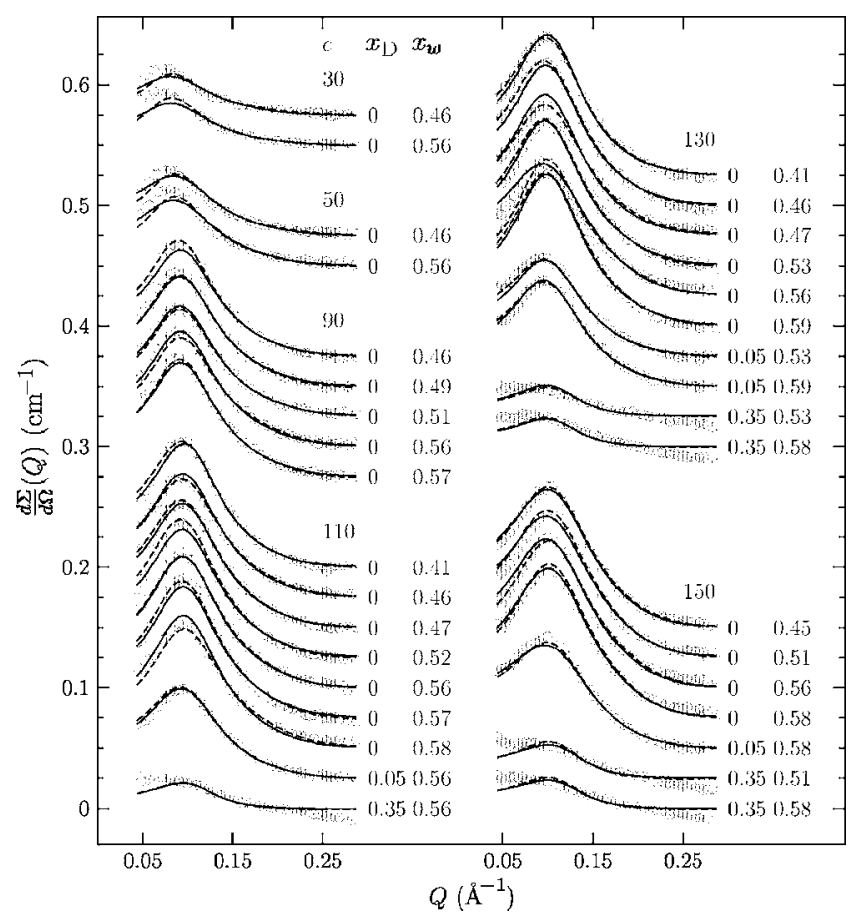

FIG. 2. SANS data obtained in the different investigated experimental conditions, as indicated. The dashed and solid lines correspond to fitting curves obtained with models 1 and 2, respectively. The symbols as in the text. For the sake of clarity, each curve has been scaled by a factor multiple of $0.025 \mathrm{~cm}^{-1}$ (indicated by minor tics) after subtraction of the corresponding background $B$ obtained using model 2 (Table I).

fraction $\left(x_{w}\right)$, and to the deuteration grade $\left(x_{\mathrm{D}}\right)$. At first, it could be observed that a satisfactory signal-to-noise ratio is obtained even in those conditions where a low scattering intensity is expected. Moreover, the differences between curves relative to different investigated experimental conditions appear significative, and confirm the expected experimental trend (see Fig. 1): increasing $x_{\mathrm{D}}$ the signal decreases, while for increasing protein concentration, the interference peak results clearer and moves to higher $Q$ values.

\section{B. Global fitting analysis}

In order to provide a unique interpretation of the whole set of experimental data, we resort to a global fitting procedure. All the scattering curves were globally analyzed by using Eq. (1), considering two different classes of fitting parameters, the first including common parameters (i.e., independent of the experimental conditions) and the second including parameters which depend on sample composition $(c$, $x_{\mathrm{D}}$, and $\left.x_{w}\right)$.

Common parameters are the protein charge $Z$ (which, at the considered $p \mathrm{H}$, is practically constant, as indicated by the lysozyme titration curve reported by Tanford and Roxby ${ }^{32}$ ), the thickness of the local domain $\delta_{l}$, the molecular volume of the water in the local domain $\nu_{w, l}$ [see Eq. (5)], and the protein volume $V_{p}$. By contrast, the parameters expected to depend on sample composition are the following. First, $J$ and $d$ factors, which describe the attractive potential [see Eq. (3)] and which have been already detected to change as a function of solvent composition in urea aqueous solutions. ${ }^{33}$ Indeed, as indicated by preliminary tests, $J$ and $d$ values were considered to be linear functions of $x_{w}$ at each $x_{\mathrm{D}}$. Second, the ionic strength of the solution $I_{S}$, which enters in the definition of the inverse Debye screening length of the Coulombic screened potential. ${ }^{34}$ In our case, lysozyme counterions and $\mathrm{HCl}$ used to adjust the $p \mathrm{H}$ mainly contribute to $I_{S}$ : as they are all proportional to $c$, different values of ionic strength were fitted for each protein concentration. Third, the solvent dielectric constant $\varepsilon$, which is involved in the Coulombic screened potential [see $u_{C}(r)$ in Eq. (2) and Ref. 25]; in this case, $\varepsilon$ has been directly calculated from $x_{w}$ and $x_{\mathrm{D}}$ according to Ref. 35. Fourth, the background $B$, which mainly accounts for the incoherent scattering of the unexchangeable lysozyme hydrogens. Fifth, the composition of the local domain, $x_{w, l}$, which was treated as an independent parameter and determined for each experimental condition.

In summary, for the global fitting procedure we considered four parameters common to all experiments $\left(Z, \delta_{l}, V_{p}\right.$, and $\nu_{w, l}$ ), two parameters singularly related to each experimental condition $\left(x_{w, l}\right.$ and $\left.B\right)$, and three parameters which linearly depend on sample composition $\left(J, d\right.$, and $\left.I_{S}\right)$. As we used six parameters to describe the linear trend of $J$ at three different deuteration grades and six other parameters for the similar estimation of $d$, and because we need to determine six values of $I_{S}$ (one for each protein concentration), the total number of fitted parameters is 92 , that can be compared to the number of analyzed SANS curves ${ }^{35}$ each one consisting of 53 experimental points.

The fitting results are shown in Fig. 2, while fitting parameters are reported in Tables I and II (indicated as model 1). In addition to self-understandable results, as background which increases with protein concentration and ionic strength which also increases with $c$ and is consistent with nominal values (Table I), other parameters deserve for some comments. From Table I it appears that $J$ and $d$ parameters, which describe the attractive potential, are smoothly dependent on solvent composition, confirming that glycerol can prevent protein-protein aggregation, as already observed for bovine pancreatic trypsin inhibitor. ${ }^{36}$ Concerning data in Table II, it can be observed that the fitted lysozyme volume $\left(V_{p}\right)$ is lower than the nominal value of $17400 \AA^{3}$ measured in water solution. ${ }^{22}$ Indeed, it has been already proven that

TABLE II. Common fitting parameters obtained by SANS data analysis with models 1 and 2 . The symbols as in the text.

\begin{tabular}{ccccccc}
\hline \hline & $\begin{array}{c}V_{p} \\
\left(\AA^{3}\right)\end{array}$ & $\begin{array}{c}\delta_{l} \\
(\AA)\end{array}$ & $\begin{array}{c}Z \\
(\mathrm{e})\end{array}$ & $\begin{array}{c}\nu_{w, l} \\
\left(\AA^{3}\right)\end{array}$ & $\begin{array}{c}\nu_{w, h} \\
\left(\AA^{3}\right)\end{array}$ & $K$ \\
\hline Model 1 & $16300 \pm 100$ & $5.9 \pm 0.2$ & $9.00 \pm 0.04$ & $29.0 \pm 0.4$ & $\ldots$ & $\ldots$ \\
Model 2 & $17060 \pm 70$ & $5.83 \pm 0.04$ & $9.1 \pm 0.3$ & $\cdots$ & $28.81 \pm 0.04$ & $1.87 \pm 0.03$ \\
\hline \hline
\end{tabular}




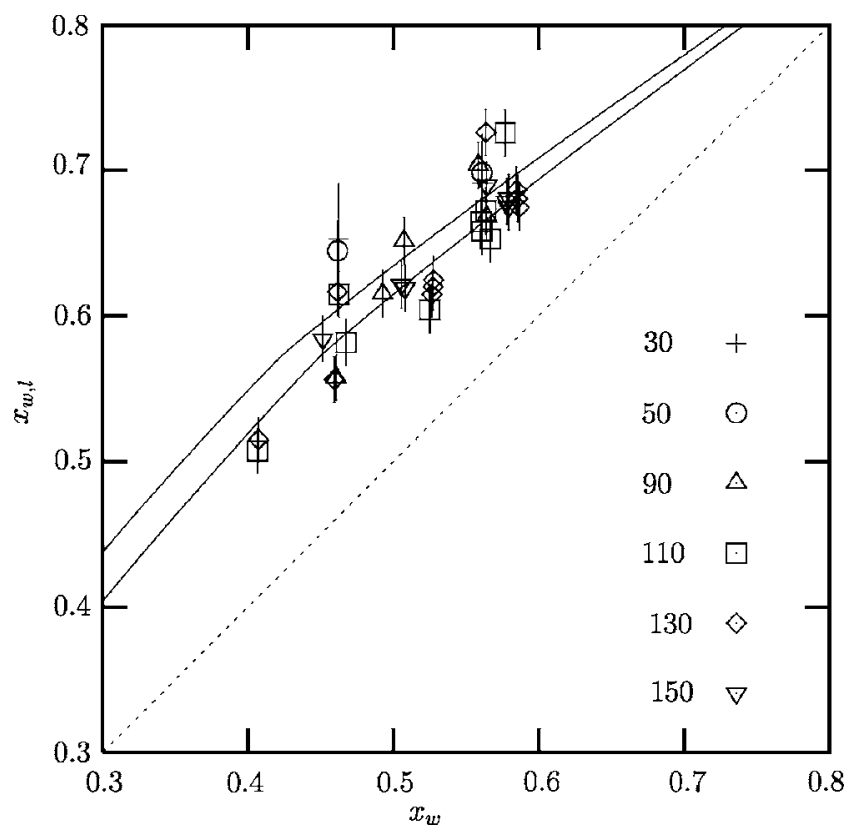

FIG. 3. Dependence on the water molar fraction in the solvent $x_{w}$ of the water molar fraction in the local domain $x_{w, l}$. The points refer to results obtained by applying model 1 , while the solid lines represent the continuous function defined in model 2 by Eq. (11), calculated with $K=1.87$ and $c$ $=30$ (bottom curve) and $c=150 \mathrm{~g} \mathrm{l}^{-1}$ (top curve). The dotted line representing the $K=1$ case is also reported.

the addition of glycerol in solution can decrease the core protein volume, probably because of the collapse of voids following the elimination of lubricant water. ${ }^{37}$ The other fitted parameters are in full agreement with previous results: the thickness of the local domain (around $6 \AA$ ) agrees with molecular dynamic simulations, which show that the first minima of the radial distribution function between glycerol and protein surfaces are at distances greater than the one corresponding to the thickness of the water hydration layer; ${ }^{31}$ the protein effective charge $Z$ agrees with the titration curve by Tanford and Roxby, ${ }^{32}$ which shows that at $p \mathrm{H}=4.5$ the charge is practically 9; the molecular volume of water in the solvation shell $\left(\nu_{w, l}\right)$ matches recent results, ${ }^{22,31}$ which indicate a water density increase in the hydration shell associated with electrostriction effects occurring at the protein surface.

Finally, the fitted water composition of the local domain, $x_{w, l}$, is reported as a function of the nominal water molar fraction of the solvent $x_{w}$ in Fig. 3. Since all determined $x_{w, l}$ are systematically larger than $x_{w}$, it follows that lysozyme is preferentially hydrated: the local domain is enriched in water with respect to bulk solvent and this effect is evident for each sample condition. Noticeable is the fact that $x_{w, l}$ continuously increases as a function of $x_{w}$.

\section{Lysozyme preferential hydration}

We will discuss extensively the preferential hydration results. Preferential hydration has been, in fact, already observed in other proteins, resulting in an estimation of the preferential binding coefficient at infinite protein dilution $\Gamma_{p j}$, a coefficient which establishes if the local domain is depleted or enriched by molecules $j$ with respect to the bulk domain. ${ }^{6,38-42}$
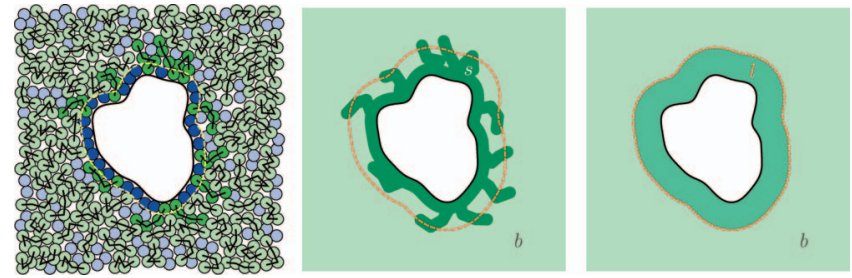

FIG. 4. (Color) Scaled representation of a solvated lysozyme molecule based on PDB structure 2LYZ (Ref. 28). The compositions of the bulk and of the first hydration layer are $x_{w, b}=0.5$ and $x_{w, h}=0.8$, respectively. Left frame: molecular aspects. The light- and dark-blue circles represent water molecules in the bulk and in the solvation layer, respectively. Four connected circles represent glycerol molecules: those in the bulk are light green, while those in contact with the protein are in dark green. The yellow line indicates the first hydration layer $(h)$, the region occupied by one shell of water molecules when the protein is fully hydrated. Central frame: solvation layer. The solvation layer $s$ is the molecular layer formed by all the molecules (water and glycerol) in contact with the protein and is represented by the dark green region. $e$ indicates the bulk phase related to the solvation shell (called "extended bulk phase"), while the red line delimits the local domain region. Right frame: local domain. The local domain is represented in dark green and corresponds to the region where are located all the molecules (water and glycerol) in contact with the protein, and where the extended bulk phase fills the gaps between the glycerol. $b$ indicates the bulk phase related to the local domain.

In the present case, $\Gamma_{p j}$ can be defined as

$$
\Gamma_{p j}=\lim _{n_{p} \rightarrow 0}\left(\frac{\partial n_{j}}{\partial n_{p}}\right)_{P, T, \mu_{j}}, j=w, g,
$$

where the indices $P, T$, and $\mu$ refer to pressure, temperature, and chemical potential of the $j$ th species, respectively. $\Gamma_{p j}$ have been experimentally determined in a few cases by a variety of thermodynamic techniques, such as dialysis equilibrium or vapor pressure osmometry. ${ }^{43}$ According to Shulgin and Ruckenstein, the preferential binding coefficient can be expressed as $\Gamma_{p j}=n_{j}\left(G_{p j}-G_{w g}\right)$, where

$$
G_{i j}=\int_{-\infty}^{\infty} d \mathbf{r}\left[g_{i j}(\mathbf{r})-1\right],
$$

and $g_{i j}(\mathbf{r})$ stands for the correlation function between species $i$ and $j$. If the composition and the extension of the local domain are known, it is straightforward to calculate $G_{p j}$ (see below), while the water/glycerol term can be approximated by considering all the activity coefficients equal to unity (see Ref. 44),

$$
G_{w g}=k_{B} T k_{T}-\left(n_{w}+n_{g}\right) \nu_{w, b} \nu_{g},
$$

being $k_{T}$ the isothermal compressibility of the mixed solvent. By writing all the number densities in terms of molar fractions and taking the limit $n_{p} \rightarrow 0$, a relationship between $\Gamma_{p j}$ and the molar fractions of water and glycerol in the bulk and in the solvation shell can be derived and the preferential binding coefficient experimentally determined. However, molar fraction data reported in Fig. 3 and Table I (model 1) cannot be used to calculate $\Gamma_{p j}$, as they are not referring to infinite dilution conditions.

To derive the composition of the protein solvation shell in the limit $n_{p} \rightarrow 0$, we then resort to the mixed solvent exchange model recently discussed by Schellman: ${ }^{45}$ the hydration process is described as a thermodynamic equilibrium, in 
which molecules in contact with the protein surface slide and exchange with molecules in the bulk, provided that one glycerol molecule replaces one water molecule only and vice versa (see the left frame of Fig. 4). ${ }^{45}$ As the characteristics of the molecular layer on the protein surface are different from those of the local domain up to now considered (see Fig. 4, central and right frames, respectively), the solvated lysozyme structure should be reconsidered.

The solvation layer ( $s$, see the central frame of Fig. 4) includes all water and glycerol molecules in contact with the protein, and then can be geometrically different from the first hydration layer $(h)$, which corresponds to the region occupied by one shell of water molecules when the protein is fully hydrated (see the left frame of Fig. 4). Nonetheless, the water molar fractions in the solvation layer and in the first hydration layer are equal, $x_{w, s}=x_{w, h}$. According to this picture, we define the number $m$ of accessible binding sites inside the first hydration layer as $m=V_{h} / \nu_{w, h}$, being $V_{h}$ the volume of first hydration layer and $\nu_{w, h}$ the volume of a single water molecule within this layer.

The process of solvent exchange over the $m$ sites of the protein surface is then described by the following thermodynamical model:

$$
g_{s}+w_{b} \rightleftharpoons g_{b}+w_{s},
$$

where $g_{b}, g_{s}$ and $w_{b}, w_{s}$ represent glycerol and water molecules in the bulk phase and in the solvation layer $(s)$, respectively. The corresponding equilibrium constant can be written as

$$
K=\frac{x_{w, h}}{1-x_{w, h}} \frac{1-x_{w, b}}{x_{w, b}} .
$$

Note that the molar fraction of water in the first hydration layer can be written as $n_{w, s} /\left(n_{p} m\right)$, since it represents the average fraction of binding sites occupied by water. Moreover, $x_{w, b}$ can be described using a different form of Eq. (6), in which all quantities referring to the local domain are replaced by those related to the first hydration layer.

To describe the form factor of the hydrated lysozyme according to the three-phase model [Eq. (4)], the following equations, which give the composition and the average molecular volume of water in the local domain from the composition of the solvation layer $\left(x_{w, s}\right)$ and the composition of the solvent filling the gaps between the glycerol molecules $\left(x_{w, b}\right)$, have been derived,

$$
\begin{aligned}
& x_{w, l}= \begin{cases}\frac{x_{w, h} m \nu_{g}-x_{w, b}\left(m \nu_{g}-V_{l}\right)+x_{w, h} x_{w, b}\left(m \nu_{w, b}-V_{h}\right)}{x_{w, h}\left(m \nu_{g}-V_{h}\right)-x_{w, b} m\left(\nu_{g}-\nu_{w, b}\right) V_{l}} & \text { for } x_{w, h}>\frac{m \nu_{g}-V_{l}}{m \nu_{g}-V_{h}} \\
x_{w, h} & \text { otherwise, }\end{cases} \\
& \nu_{w, l}=\frac{x_{w, h}\left(x_{w, b}-x_{w, l}\right)}{x_{w, l}\left(x_{w, b}-x_{w, h}\right)}\left(\nu_{w, h}-\nu_{w, b}\right)+\nu_{w, b} .
\end{aligned}
$$

Through Eq. (10), $x_{w, l}$ becomes a direct function of the equilibrium constant $K$. Moreover, incorporating Eqs. (11) and (12) in Eq. (5), also the scattering length densities $\rho_{l}$ and $\rho_{b}$ result directly bound to solvation features. Therefore, a new global analysis of all experimental data has been performed to estimate the composition of the local domain by fitting the value of the thermodynamic constant $K$.

Global fitting results are shown in Fig. 2 and in Tables I and II (indicated as model 2). At first, it should be observed that fitted parameters are very similar to those obtained in the previous analysis. However, the main result is that the $K$ value of $1.87 \pm 0.03$ (Table II) confirms that lysozyme dissolved in water/glycerol mixed solvents is preferentially hydrated.

More interesting, from the estimated value of $K$, the preferential binding coefficient can be calculated. In fact, as the composition of the hydration layer is known, the $G_{p j}$ term can be written as

$$
G_{p j}=n_{j, s} /\left(n_{p} n_{j}\right)-V_{p}-V_{s}, \quad j=w, g,
$$

where $V_{s}=m\left(\nu_{w, h} x_{w, h}+\nu_{g} x_{g, h}\right)$ is the volume of the solvation layer. The following relationship between $\Gamma_{p j}, x_{w}$, and Schellman's constant $K$ is finally derived:

$$
\begin{aligned}
\Gamma_{p j}= & m x_{j, h}+\frac{x_{j}}{\nu_{w, b} x_{w}+\nu_{g} x_{g}} \\
& \times\left[\frac{\nu_{w, b} \nu_{g}}{\nu_{w, b} x_{w}+\nu_{g} x_{g}}-V_{p}-m\left(\nu_{w, h} x_{w, h}+\nu_{g} x_{g, h}\right)-k_{B} T k_{T}\right] .
\end{aligned}
$$

Here the water molar fraction $x_{w, h}$ represents the limit value of Eq. (11) for $n_{p} \rightarrow 0$,

$$
x_{w, h}=\frac{x_{w} K}{x_{w}(K-1)+1} .
$$

The calculated $\Gamma_{p j}$ values are reported in Fig. 5 and compared with data reported by Gekko and Timasheff ${ }^{6}$ for ribonuclease $A$ in water/glycerol mixed solvent at low glycerol content. Even if the two proteins are different, and even if the 

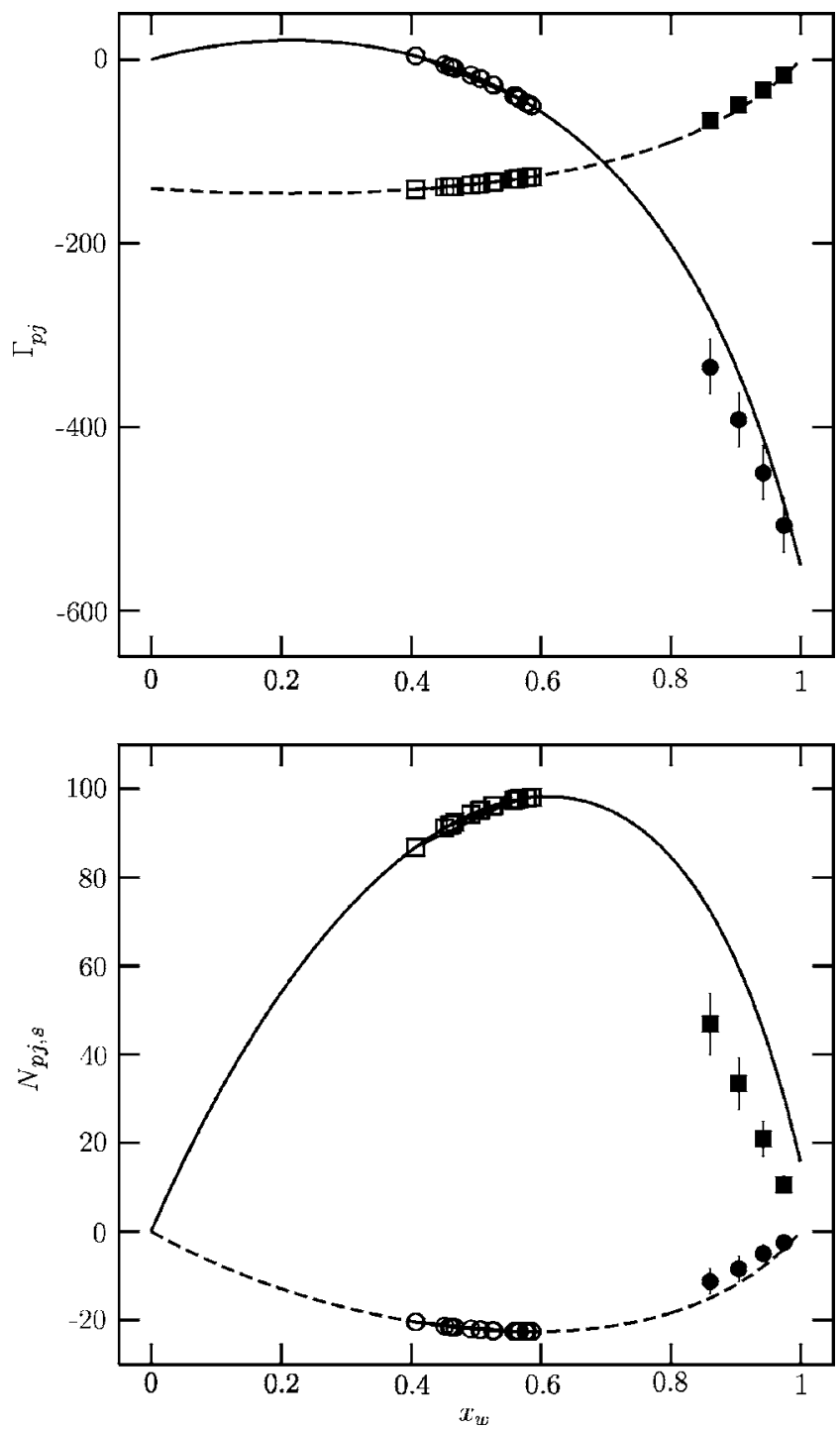

FIG. 5. Dependence on the water molar fraction in the solvent $x_{w}$ of the preferential binding coefficient $\Gamma_{p j}$ (top frame) and of the excess solvation number $N_{p j, s}$ of the solvation layer (bottom frame). The curves have been calculated with Eqs. (14) and (16) using the parameters obtained by model 2 fitting analysis: the solid and dotted lines refer to water $(j=w)$ and glycerol $(j=g)$, respectively. The open symbols indicate all the investigated experimental points. The close symbols refer to data relative to the ribonuclease $A$, as reported in Refs. 6 and 44.

investigated concentrations are very different, the agreement between the two sets of data is very satisfactory.

The analysis has been also extended to the calculation of the excess solvation number of species $j, N_{p j}=n_{j} G_{p j}$, which represents the number of displaced molecules $j$ when a protein molecule is introduced into the mixed solvent. ${ }^{46}$ This change is comprehensive of two factors: the inaccessibility of solvent molecules to protein volume and the solventsolvent interactions arising when the protein is introduced into the system (mainly due to solvent reorganization). We calculated the excess solvation number in the solvation layer $N_{p j, s}=N_{p j}+n_{j} V_{p}$, which is the difference between the number of molecules of kind $j$ in the solvation layer and the number of molecules of the same kind in an equal volume belonging to the extended bulk phase, ${ }^{46,47}$

$$
N_{p j, s}=m\left(x_{j, h}-x_{j} \frac{\nu_{w, h} x_{w, h}+\nu_{g} x_{g, h}}{\nu_{w, b} x_{w}+\nu_{g} x_{g}}\right) .
$$

In Fig. 5 the obtained values are reported as a function of water molar fraction in the solvent: it is even clearer that the presence of glycerol in the solvent enhances the amount of water in the solvation layer. The bell-shaped form of the two curves, which have been derived using Eqs. (14) and (16), should be underlined. The two curves completely reproduce the experimentally observed behaviors. It can be concluded that a similar mechanism underlies the preferential hydration process, both in the presence of small and large amounts of glycerol.

The $N_{p w, s}$ curve also predicts the existence of a concentration region of maximal hydration, caused by the right balance between the enhanced hydration due to the increased water affinity at the protein surface and the availability of water in the mixed solution at higher glycerol concentration. This phenomenon is particularly interesting, as can be related to the changes in protein stability already observed in the presence of cosolvents. ${ }^{13-15}$ In particular, it can be anticipated that thermal stability of lysozyme measured as a function of glycerol content in the same solvent shows a very similar trend. ${ }^{48}$

\section{CONCLUSION}

The original aspect of this study is that a quantitative characterization of the protein-solvent interface of lysozyme dissolved in water/glycerol mixtures results from the global fit analysis of the 35 SANS curves obtained in different experimental conditions.

In particular, four results can be underlined. First, the measured thickness of the local domain is in full agreement with molecular dynamic simulations, which show that the first minima of the radial distribution function between glycerol and protein surface are at distances greater than that corresponding to the thickness of the usual water hydration layer. ${ }^{31}$ Second, the molecular volume of the water in the first hydration layer is about $4 \%$ smaller than the volume of pure water, in full agreement with previous SAXS/SANS results. ${ }^{22}$ Third, the attractive term of the protein-protein interaction potential decreases by increasing glycerol concentration in the mixed solvent, confirming that glycerol prevents protein-protein aggregation. Fourth, the preferential hydration of the lysozyme has been confirmed at all the investigated experimental conditions, especially in the glycerol rich region, not accessible to vapor pressure osmometry and density experiments ${ }^{6}$ because of high mixture viscosity. By using a model of solvent exchange between the lysozyme solvation shell and the bulk, the preferential binding coefficient and the excess solvation number have been estimated. As a main result, the curve describes the excess solvation number as a function of the solvent.

\footnotetext{
${ }^{1}$ C. Tanford, Physical Chemistry of Macromolecules (Wiley, New York, 1961)

${ }^{2}$ M. C. Bellissent-Funel, Hydration Processes in Biology (IOS, Amsterdam, 1999).

${ }^{3}$ K. Gekko, J. Biochem. (Tokyo) 91, 1197 (1982).

${ }^{4}$ P. H. Yancey, M. E. Clark, S. C. Hand, R. D. Bowlus, and G. N. Somero,
} 
Science 217, 1214 (1982).

${ }^{5}$ Q. Zou, B. J. Bennion, V. Daggett, and K. P. Murphy, J. Am. Chem. Soc. 124, 1192 (2002).

${ }^{6}$ K. Gekko and S. N. Timasheff, Biochemistry 20, 4667 (1981).

${ }^{7}$ M. S. Lehmann and G. Zaccai, Biochemistry 23, 1939 (1984).

${ }^{8}$ E. S. Courtenay, M. W. Capp, C. F. Anderson, and M. T. Record, Jr., Biochemistry 39, 4455 (2000).

${ }^{9}$ S. N. Timasheff, Annu. Rev. Biophys. Biomol. Struct. 22, 67 (1993).

${ }^{10}$ T. Knubovets, J. J. Osterhout, P. J. Connolly, and A. M. Klibanov, Proc. Natl. Acad. Sci. U.S.A. 96, 1262 (1999).

${ }^{11}$ K. Griebenow and A. M. Klibanov, Proc. Natl. Acad. Sci. U.S.A. 92, 10969 (1995).

${ }^{12}$ R. V. Rariy and A. M. Klibanov, Proc. Natl. Acad. Sci. U.S.A. 94, 13520 (1997).

${ }^{13}$ A. Paciaroni, S. Cinelli, and G. Onori, Biophys. J. 83, 1157 (2002).

${ }^{14}$ A. Paciaroni, A. Orecchini, S. Cinelli, G. Onori, R. E. Lechner, and J. Pieper, J. Chem. Phys. 292, 397 (2003).

${ }^{15}$ S. Cinelli, A. De Francesco, G. Onori, and A. Paciaroni, Phys. Chem. Chem. Phys. 6, 3591 (2004).

${ }^{16}$ D. Sprous, M. A. Young, and D. L. Beveridge, J. Phys. Chem. B 102, 4658 (1998)

${ }^{17}$ J. Tirado-Rives, M. Orozco, and W. L. Jorgensen, Biochemistry 36, 7313 (1997)

${ }^{18}$ L. A. Feigin and D. I. Svergun, Structure Analysis by Small-Angle X-Ray, Neutron Scattering (Plenum, New York, 1987).

${ }^{19}$ F. Spinozzi, F. Carsughi, P. Mariani, C. V. Teixeira, and L. Q. Amaral, J. Appl. Crystallogr. 33, 556 (2000).

${ }^{20}$ F. Spinozzi, D. Gazzillo, A. Giacometti, P. Mariani, and F. Carsughi, Biophys. J. 82, 2165 (2002).

${ }^{21}$ B. Jacrot, Rep. Prog. Phys. 39, 911 (1976)

${ }^{22}$ D. Svergun, S. Richard, M. H. J. Koch, Z. Sayers, S. Kuprin, and G. Zaccai, Proc. Natl. Acad. Sci. U.S.A. 95, 2267 (1998).

${ }^{23}$ M. G. Ortore, F. Spinozzi, C. Carsughi, P. Mariani, M. Bonetti, and G. Onori, Chem. Phys. Lett. 418, 338 (2005).
${ }^{24}$ F. Spinozzi, E. Maccioni, C. V. Teixeira, H. Amenitsch, R. Favilla, M. Goldoni, P. Di Muro, B. Salvato, P. Mariani, and M. Beltramini, Biophys. J. 85, 2661 (2003)

${ }^{25}$ J. Narayanan and X. Y. Liu, Biophys. J. 84, 523 (2003).

${ }^{26}$ A. J. Dianoux and G. Lander, Neutron Data Booklet (OCP Science, Grenoble, France, 2003).

${ }^{27}$ D. R. Lide, Handbook of Chemistry and Physics, 77th ed. (CRC, Cleveland, OH, 1996).

${ }^{28}$ R. Diamond, J. Mol. Biol. 82, 371 (1974).

${ }^{29}$ F. Spinozzi, F. Carsughi, P. Mariani, L. Saturni, S. Bernstorff, S. Cinelli, and G. Onori, J. Phys. Chem. 111, 3822 (2007).

${ }^{30}$ S. Tang, R. E. Karen, and V. A. Bloomfield, Biophys. J. 82, 2876 (2002).

${ }^{31}$ B. M. Baynes and B. L. Trout, J. Phys. Chem. B 107, 14058 (2003).

${ }^{32}$ C. Tanford and R. Roxyb, Biochemistry 11, 2192 (1972).

${ }^{33}$ M. Niebuhr and M. H. J. Koch, Biophys. J. 89, 1978 (2005).

${ }^{34}$ E. J. W. Vervey and J. T. G. Overbeek, Theory of the Stability of Lyophobic Colloids (Elsevier, Amsterdam, 1948).

${ }^{35}$ T. Erdey-Grz, Transport Phenomena in Aqueous Solutions (Hilger, London, 1974).

${ }^{36}$ M. Farnum and C. Zukoski, Biophys. J. 76, 2716 (1999).

${ }^{37}$ A. Priev, A. Almagor, S. Yedgar, and B. Gavish, Biochemistry 35, 2061 (1996).

${ }^{38}$ S. N. Timasheff, Adv. Protein Chem. 51, 355 (1998).

${ }^{39}$ S. Shimizu, J. Chem. Phys. 120, 4989 (2004).

${ }^{40}$ G. Xie and S. N. Timasheff, Biophys. Chem. 64, 25 (1997).

${ }^{41}$ I. L. Shulgin and E. Ruckenstein, Biophys. J. 90, 704 (2006).

${ }^{42}$ S. Shimizu and N. Matubayasi, Chem. Phys. Lett. 420, 518 (2006).

${ }^{43}$ W. Zhang, M. W. Capp, J. P. Bond, C. F. Anderson, and M. T. Record, Jr., Biochemistry 35, 10506 (1996).

${ }^{44}$ I. L. Shulgin and E. Ruckenstein, J. Chem. Phys. 123, 054909 (2005).

${ }^{45}$ J. A. Schellmann, Biophys. J. 85, 108 (2003).

${ }^{46}$ S. Shimizu, Proc. Natl. Acad. Sci. U.S.A. 101, 1195 (2004).

${ }^{47}$ S. Shimizu and D. J. Smith, J. Chem. Phys. 121, 1148 (2004).

${ }^{48} \mathrm{G}$. Onori (private communication). 